\title{
INTELLIGENT DECISION SUPPORT SYSTEM FOR ADAPTIVE SLICING IN RPT PROCESS
}

\author{
RASOVIC, N[ebojsa]; KALJUN, J[asmin] \& OBAD, M[ilenko]
}

\begin{abstract}
A lot of software solutions for design analysis are based on numerical approaches, but they mainly cannot provide any kind of expert advice during the process. This paper discusses a framework for intelligent decision support for fully design analysis of the CAD model, taking into consideration their functional, aesthetic and ergonomic features. A knowledge base is developed and as such contains mentioned features, specific for adaptive slicing in rapid prototyping process. The CAD model is evaluated by using weighting factors algorithm, which is crucial in determining of the layer thickness in the RPT process. The handle of electrical device is used in the case study to build intelligent decision support system in order to calculate layer thickness.

Keywords: intelligent decision support, knowledge base, adaptive slicing.
\end{abstract}

\section{INTRODUCTION}

Rapid prototyping production is limited between surface quality and production time of the model. The thinner layer thickness leads to an increase production time, while the thicker layer thickness creates poor surface quality of the model. This theory is most relevant for constant layer thickness[1].

The model accuracy not only depends upon the layer thickness but also the geometrical complexity. It is important to note that the value of the layer thickness is between $t_{\min }$ and $t_{\max }$ [2]. This range is crucial to find a compromise between two mentioned criteria. However, that range is limited by fabrication capabilities of the specific layer manufacturing process.

In order to find an optimal layer thickness, a model could be evaluated in terms of its functional, aesthetic and ergonomic features. This paper discusses possible framework which propose an optimal layer thickness using intelligent decision support system.

There are many software solutions for fully design analysis, including concept evaluation, but they cannot provide any kind of advice in certain phase of design process. Main goal of this research is to find an optimal concept using weighting factor which has a key role when calculate final layer thickness.

In order to solve this task, the prototype of the intelligent advisory system is briefly presented. This intelligent module needs to provide multiple solutions at any time during the userös decision making process. Module takes into consideration main features of the model and based on that make decision for optimal thickness. This approach could be taken for increasing RP manufacturability.

\section{INTELLIGENT SUPPORT BASED ON THE CAD MODEL FEATURES}

Different approaches have been taken with the aim to optimize the design of the product. Many of them [3] propose multi parameter optimization using weighting factors. However, weighting factors are usually applied in selection (decision making) process (figure 1). The CAD model is under consideration in terms of his basic characteristic. After that, model is divided into segments with certain values of the appropriate weighting factors. These results are written into a current case data base in order to be used for further calculation of layer thickness.

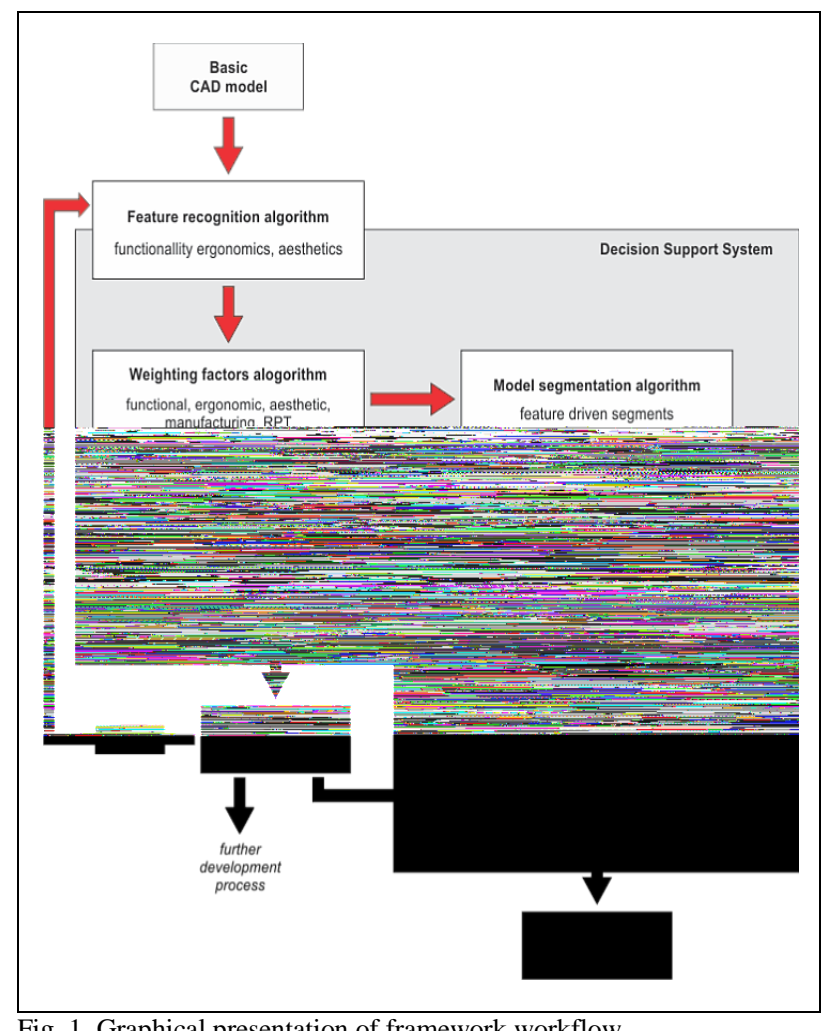

Fig. 1. Graphical presentation of framework workflow

Knowledge-based decision support for adaptive slicing is based on the series of rules which define the procedure for layer thickness selection. In expert system techology, each of the expert's rules is a heuristic. The combination of all the heuristics allows the overall decision making problem to be solved. Main features of the CAD model are incorporated in the intelligent module and they represent influential parameters in process decision (figure 2). 


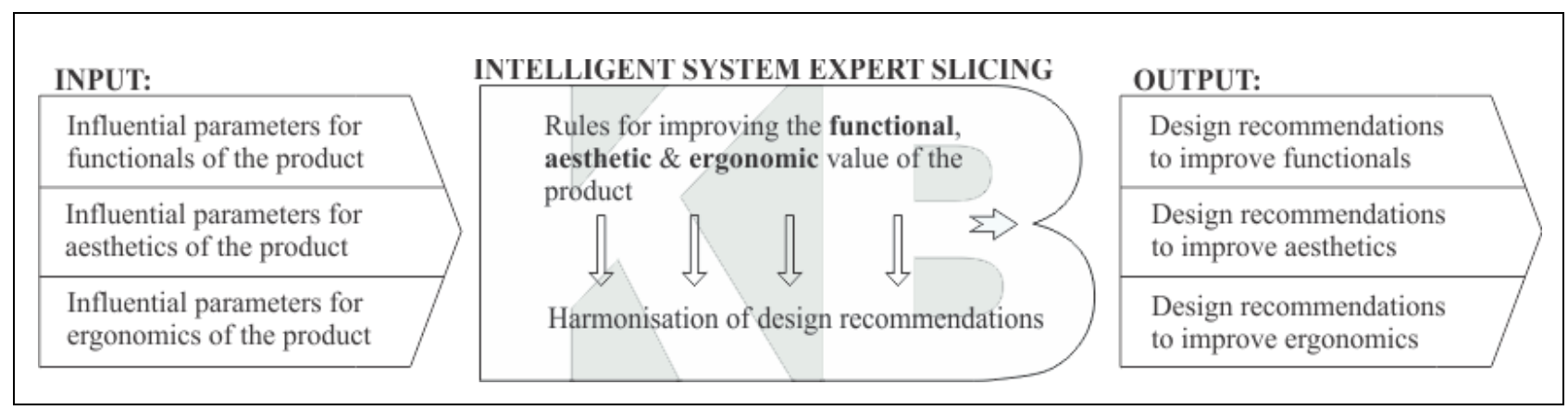

Fig. 2.Basic structure of the intelligent advisory system expert slicing $[4,5]$

It is very important to note that the intelligent decision support system óExpert Slicingô is still a research prototype and, as such, the subject of intensive development. The main focus is on the model evaluation in term of his functional, aesthetic and ergonomic requirements in manufacturing process. Knowledge base is expandable, but it was decided to limit research on design of the handle electrical device. When developing Expert Slicing, a development environment named Exsys Corvid [6] has been used. The Corvid enables developer to build the knowledge base using natural language. User interface is built simultaneously with knowledge base. The Corvid provides developer with configurable inference engine. The prototype is built as java applet to be used online. System can be integrated with personalization of software allowing it that automatically provides some of the data, the system will need.

Different classes are connected with various attributes and their values in head of the rule in order to describe case specific situation, as for example, model position in RP machine (a rule from ExsysCorvid):

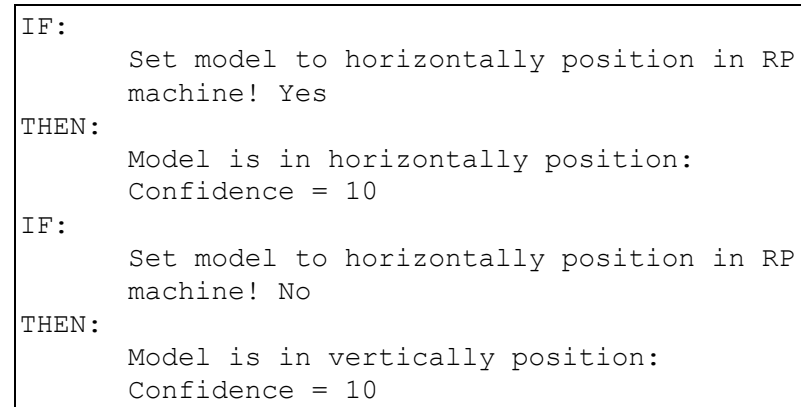

Alg. 1. Rule from ExsysCorvid

\subsection{Configuration and division of the CAD model}

The CAD model is sometimes used only for visualization and correctness of a model design, so its aesthetic value is being evaluated. However, a model can also be used for testing certain functional and ergonomic characteristics, but it is quite often that aesthetic, functional and ergonomic features must be taken into consideration on the same product. These thoughts lead to the point that each model should be evaluated according to its aesthetic, functional and ergonomic demands concerning quality of surface on certain parts of the model.

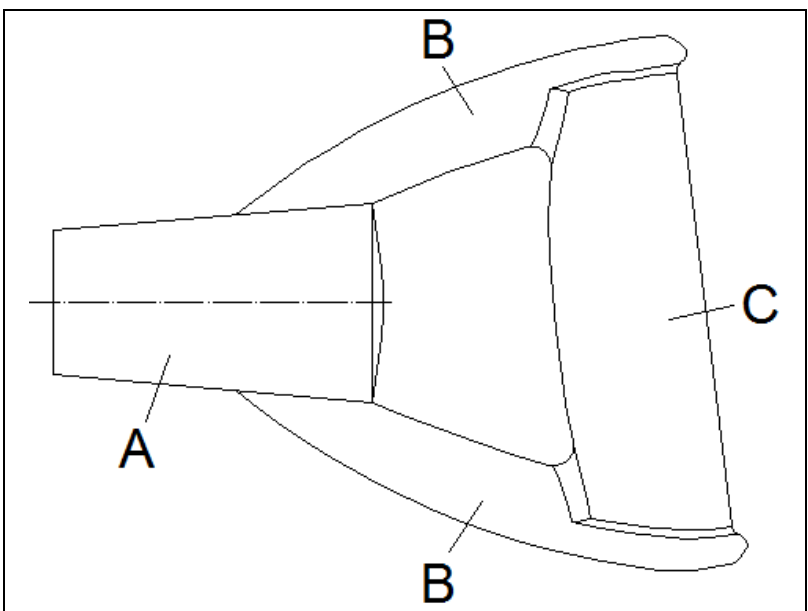

Fig. 3. The CAD model ofelectrical device handle

Figure 3 shows a handle of an electrical device. Three specific parts of the handle are marked with letters A, B and C. The first part marked with letter A presents the central part which connects a handle with other part of the machine. This part is important for functionality of the machine and it is certain that its functionality is very important. Considering the way of joining with the machine this part of the model will require more quality manufacturing.

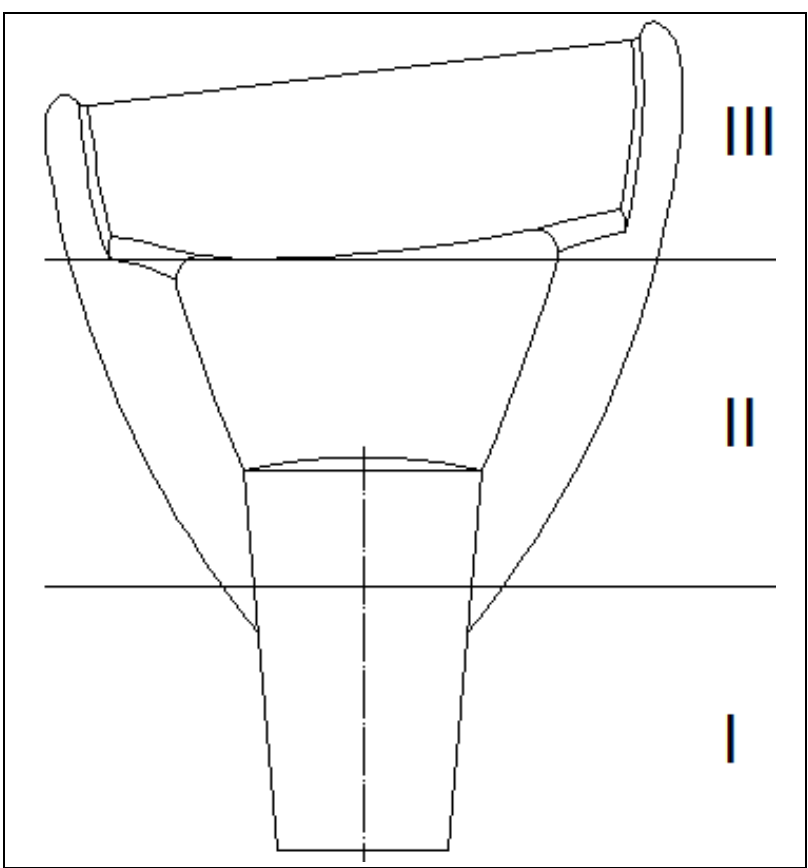

Fig. 4. Division of the CAD model into segments (zones) 
Parts of the model marked with letter B are side girders of the handle which is marked with letter $\mathrm{C}$. Those side girders do not require special manufacturing quality neither in functional nor in aesthetic sense. The handle itself will require significantly higher quality.

Regarding to the previously mentioned aesthetic, functional and ergonomic specificity of certain parts, the model is rotated into the best position for RP manufacturing, as it is shown in the figure 4. The goal is to enable that different optimal heights of layers in zones I, II and III are defined.

Because of this it is useful that the óweighting surface factorsô (w) are joined to the zones, when it is about surface quality i.e. the layer height of RP model. Those weighting factors are defined on the basis of aesthetic, functional and ergonomic demands in a model surface quality. After that, they are used in calculation of layer height, so the calculated height itself could be corrected and with the aim to obtain final height $t_{\text {final }}$.

\section{RESULTS IMPLEMENTATION USING EXPERT SLICING}

Usefulness of advice provided by intelligent system, in the first place depends on the input influential parameters. For this case, input parameters are defined by designer to carry out the division of the CAD model into segments. These rules in the intelligent module Expert Slicing appear as shown below:

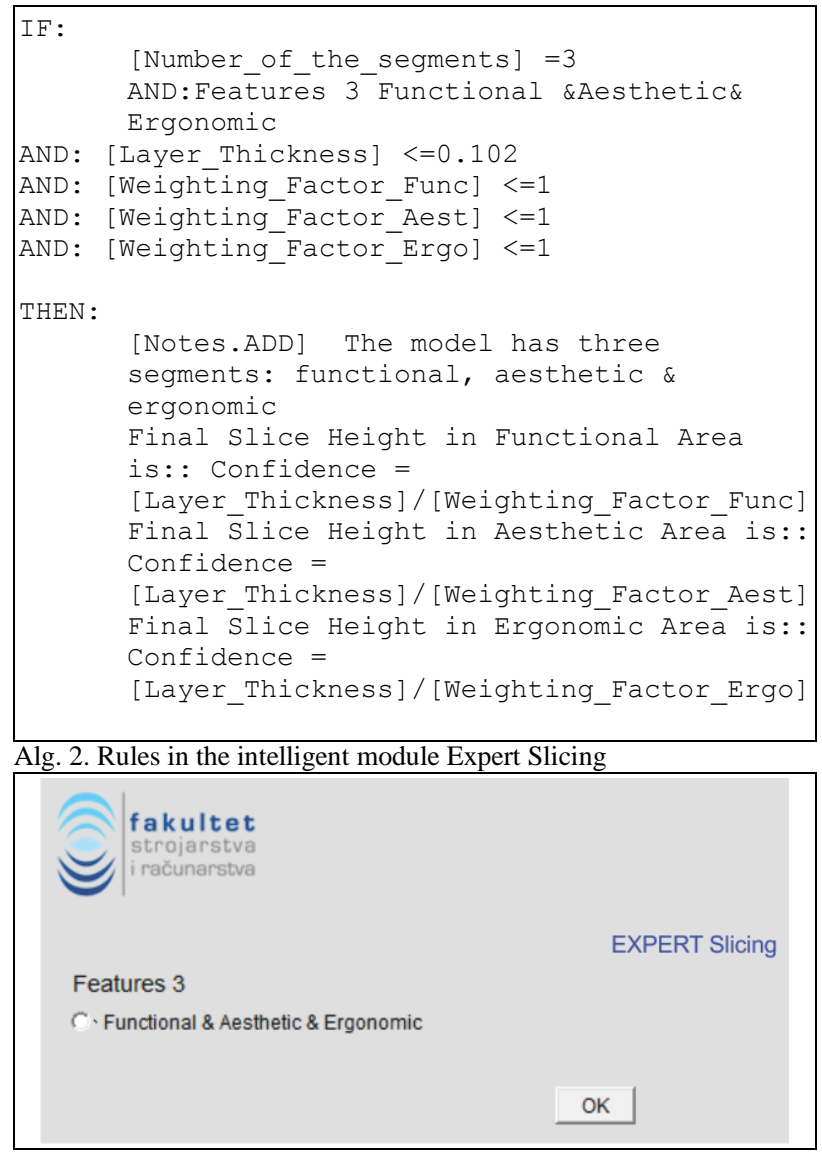

Fig 5. Recommended features by Expert Slicing
In order to show appropriate values of the weighting factors, related to the electrical device handle, these values are given in the table 1 .

\begin{tabular}{|c|c|c|c|c|c|}
\hline \multicolumn{6}{|c|}{ Functionalweightingfactors } \\
\hline & v1 & v2 & v3 & Ù1 & wi \\
\hline v1 & & 1 & 1 & 2 & 1 \\
\hline v2 & 2 & & 0 & 0 & 0 \\
\hline v3 & 2 & 0 & & 0 & 0 \\
\hline \multicolumn{4}{|c|}{ Ù } & 2 & 1 \\
\hline \multicolumn{6}{|c|}{ Aestheticweightingfactors } \\
\hline & $\mathrm{v} 1$ & v2 & v3 & Ù1 & wi \\
\hline v1 & & 2 & 2 & 0 & 0 \\
\hline v2 & 1 & & 0 & 1 & 0,5 \\
\hline v3 & 1 & 0 & & 1 & 0,5 \\
\hline & & & & 2 & 1 \\
\hline \multicolumn{6}{|c|}{ Ergonomicweightingfactors } \\
\hline & v1 & v2 & v3 & Ù1 & wi \\
\hline $\mathrm{v} 1$ & & 0 & 2 & 0 & 0 \\
\hline $\mathrm{v} 2$ & 0 & & 2 & 0 & 0 \\
\hline v3 & 1 & 1 & & 2 & 1 \\
\hline \multicolumn{4}{|c|}{ Ù } & 2 & 1 \\
\hline
\end{tabular}

Tab. 1. The values of weighting factors by the zones

When defining final weighting factor for each zone it is necessary to take bigger of the three offered.

\begin{tabular}{|c|c|}
\hline & zone I \\
\hline $\mathrm{w}_{\text {fun. }}$ & 1(high quality) \\
\hline $\mathrm{w}_{\text {est. }}$ & 0 (low quality) \\
\hline \multirow[t]{2}{*}{$\mathrm{w}_{\text {erg. }}$} & 0(low quality) \\
\hline & zone II \\
\hline $\mathrm{w}_{\text {fun. }}$ & 0(low quality) \\
\hline $\mathrm{w}_{\text {est. }}$ & 0,5 (medium quality) \\
\hline \multirow[t]{2}{*}{$\mathrm{w}_{\text {erg. }}$} & 0 (low quality) \\
\hline & zone III \\
\hline $\mathrm{w}_{\text {fun. }}$ & O(low quality) \\
\hline $\mathrm{w}_{\text {est. }}$ & 0,5 (medium quality) \\
\hline $\mathbf{W}_{\text {erg. }}$ & 1(high quality) \\
\hline
\end{tabular}

Tab. 2. Obtained values of weighting factors by model zones

The procedure to define weighting factors ówô can be different depending on complexity of the model and different aesthetic, functional and ergonomic demands. Expert slicing use one of possible ways to arrange weighting factors in zones I, II and III, taking into account aesthetic, functional and ergonomic values of the model. The method by which preference is defined on the basis of comparing a segment (zones) of the CAD model to all other segments of the mentioned model will be used to obtain weighting factors. If the segment which is compared has the preference, the value 1 is written, if the segment to which it is compared has the preference the value 2 is written, and if the compared segments are of the same importance, than 0 is written [7-11]. Relations between segments of the electrical device handle model and calculated value of weighting factors for each variant are shown in table 1 .

It is important to note, that the intelligent system Expert Slicing, is in development stage, and is still not able to make decisions in determining preference for allocation weighting factors. 


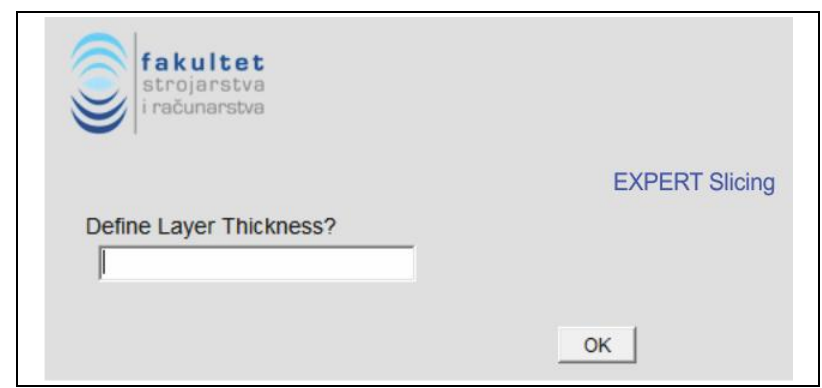

Fig. 6.Recommended layer thickness by Expert Slicing

Expert Slicing allows that the layer thickness can be defferent for all three features of the model. However, the methodology which is presented in this paper does not provide direct changing of the layer thickness, but it depends on the value of the weighting factor. Layer height is limited up to $0.102 \mathrm{~mm}$, which is upper limit for 3D Print technology. If entered value is higher than the allowed one, Expert Slicing will repeat the request for admission. The lower limit is $0.089 \mathrm{~mm}$.

After defining layer thickness, application offers form to fill appropriate weighting factor. For each feature (segment of the model), user must define weighting factor, in this case, there are three values. His value is limited between 0 and 1 . It should be noted that value which is closer 1 , has higher priority in surface quality production, while the opposite case has lower priority in surface quality production.

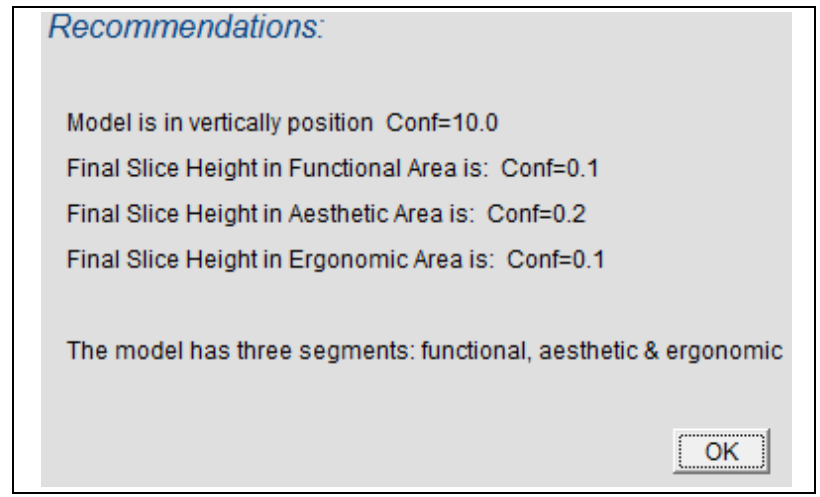

Fig. 7. Recommended results for the layer height by Expert Slicing

Intelligent system in making decision offers possible solutions (recommendations) in a few individual steps. For electical device handle, the best position for manufacturing is verticall position.

Functional and ergonomic area is very important from manufacturing point, and value of the weighting factor is high, which colud be seen from the final slice height and amounts 0.1. It is seen (figure 7.) that final layer height in aesthetic area (zone II) is twice higher then size of the calculated value. If that calculated height is higher than the highest possible height that can be done on RP machine, than it is corrected to that height. In that way, the model aesthetic area (zone II) can be made two times faster regardless the calculated height of the layer. Finally, application reminds designer, that the electrical device handle has three features.

Future research plan leads to the improvement and implementation of the proposed intelligent system and further development of this method.

\section{CONCLUSION}

Decision making is the process of developing and analyzing alternatives to make a decision. Most decisions are made in response to a problem. Although each decision is specific in certain domain, it can find common principles for making better decissions. In the initial phase, the designer has to take decisions, influenced by various parameters, according to the available data. One of the crucial decisions is consideration model from the perspective of its most important characteristics. Modern software solutions assist engineers during product development, but there are still limitations at offering some expert advice or recommendation at decission making process. Designerös aim is to have as many optimal solutions as possible, meaning results on diverse queries from designing a product shape.

The functional, aesthetic and ergonomic features of the CAD model are a vital and complex part of the product design process. Regarding the complexity of the model surface and his manufacturing, an intelligent support to adaptive slicing in the form of advisory decision support system is proposed. There are many proposed algorithms in the field of adaptive slicing, but the majority of them are not focused on the main features of a certain model.

It is very hard to satisfy all of the attributes that define a single product, but with proposed intelligent support, it is possible to find a compromise between production time and quality, which is obtained in the manufactured item.

Expert systems developed by Exsys Corvid directly deliver knowledge, advice and recommendation, rather than just information. Development environment of Exsys Corvid is time limited up to 30 days.

\section{REFERENCES}

[1] Ron, J. \& Herbert, H., (1995) Direct slicing of CAD models for rapid prototyping. Rapid Prototyping Journal. 1(2): p. 4.

[2] Tyberg, J., (1998) Local adaptive slicing for layered manufacturing. University Libraries, Virginia Polytechnic Institute and State University: Blacksburg, Va.

[3] Steven, G.P., Li, Q. \& Xie, Y.M., (2002) Multicriteria optimization that minimizes maximum stress and maximizes stiffness. Computers \&amp; Structures. 80(27ñ30): p. 2433-2448.

[4] Dolǵak, B. \& Novak, M., (2011) Intelligent decision support for structural design analysis. Advanced Engineering Informatics. 25(2): p. 330-340.

[5] Kaljun, J. \& Dolǵak, B., (2012) Ergonomic design knowledge built in the intelligent decision support system. International Journal of Industrial Ergonomics. 42(1): p. 162-171.

[6] Awad, E.M., (2003) Building knowledge automation expert systems : with Exsys CORVID, Albuquerque, N.M.: EXSYS Inc.

[7] Haik, Y., (2003) Engineering design process, [South Melbourne, Victoria], Australia; Pacific Grove, CA: Thomson/Brooks/Cole.

[8] Ullman, D.G., (2010) The mechanical design process, Boston: McGraw-Hill Higher Education.

[9] Gibson, I., Rosen, D.W. \& Stucker, B., (2010) Additive manufacturing technologies rapid prototyping to direct digital manufacturing, New York: Springer.

[10] Crowson, R., (2006) Product design and factory development, Boca Raton, Fla.: Taylor \& Francis.

[11] Raǵovil, N. \& Obad, M., (2012) Adaptive slicing in 3D printing process. Proceedings of The 7th International Symposium on Machine And Industrial Design In Mechanical Engineering KOD 2012, ISBN 978-86-7892-399-9, Balatonfüred, Hungary, Faculty of Technical Sciences, Novi Sad, p. 243-246. 